\title{
Recursive Identification of Switched ARX Models with Unknown Number of Models and Unknown Orders
}

\author{
Yasmin Hashambhoy
}

\author{
René Vidal
}

\begin{abstract}
We consider the problem of recursively identifying the parameters of a switched ARX (SARX) model from input/output data under the assumption that the number of models, the model orders and the switching sequence are unknown. Our approach exploits the fact that applying a polynomial embedding to the input/output data leads to a lifted ARX model whose dynamics are linear on the so-called hybrid model parameters and independent of the switching sequence. In principle, one can use a standard recursive algorithm to identify such hybrid parameters. However, when the number of models and the model orders are unknown the embedded regressors may not be persistently exciting, hence the estimates of the hybrid parameters may not converge exponentially to a constant vector. Nevertheless, we show that these estimates still converge to a vector that depends continuously on the initial condition. By identifying the hybrid model parameters starting from two different initial conditions, we show that one can build two homogeneous polynomials whose derivatives at a regressor give an estimate of the parameters of the ARX model generating that regressor. After properly enforcing some of the entries of the hybrid model parameters to be zero, such estimates are shown to converge exponentially to the true ARX model parameters under suitable persistence of excitation conditions on the input/output data. Although our algorithm is designed for the case of perfect input/output data, our experiments also show its performance with noisy data.
\end{abstract}

\section{INTRODUCTION}

Consider a discrete-time Switched Auto Regressive eXogenous (SARX) system whose dynamics are given by

$$
y_{t}=\sum_{j=1}^{n_{a}\left(\lambda_{t}\right)} a_{j}\left(\lambda_{t}\right) y_{t-j}+\sum_{j=1}^{n_{c}\left(\lambda_{t}\right)} c_{j}\left(\lambda_{t}\right) u_{t-j}
$$

where $u_{t} \in \mathbb{R}$ is the input, $y_{t} \in \mathbb{R}$ is the output, and $n_{a}(i)$, $\left\{a_{\ell}(i)\right\}_{\ell=1}^{n_{a}(i)}$ and $\left\{c_{\ell}(i)\right\}_{\ell=1}^{n_{c}(i)}$ are, respectively, the orders and the model parameters of the $i$ th ARX model for $i=1, \ldots, n$. The discrete state or mode $\lambda_{t}$ is assumed to be a deterministic but unknown sequence that can take a finite number of possible values: $\lambda: \mathbb{Z} \rightarrow\{1,2, \ldots, n\}$.

The purpose of this paper is to characterize sufficient conditions on the input and switching sequences and develop a recursive algorithm for solving the following problem:

Problem 1 (Identification of Switched ARX Systems): Given input/output data $\left\{u_{t}, y_{t}\right\}_{t=0}^{\infty}$ generated by an SARX system such as (1), identify the number of ARX systems $n$, the orders of each ARX system $\left\{n_{a}(i), n_{c}(i)\right\}_{i=1}^{n}$, and the system parameters $\left\{a_{j}(i)\right\}_{j=1}^{n_{a}(i)}$ and $\left\{c_{j}(i)\right\}_{j=1}^{n_{c}(i)}$.

This work has been funded by Johns Hopkins Whiting School of Engineering startup funds and by grant NSF EHS-0509101

The authors are with the Center for Imaging Science, Department of Biomedical Engineering, Johns Hopkins University, 3400 N. Charles St., Baltimore, MD, 21218, USA \{yasmin,rvidal $@$ cis.jhu.edu
Our previous work [9] showed that when the orders of the constituents ARX systems are equal and known and the number of models is also known, Problem 1 can be solved by applying a polynomial embedding to the input/output data. This embedding leads to a new lifted dynamical model whose dynamics are linear on the so-called hybrid model parameters $\boldsymbol{h}$ and independent of the switching sequence. Therefore, one can use a standard recursive identifier, which yields an exponentially convergent estimate of $\boldsymbol{h}$ under suitable persistence of excitation conditions on the input/output data. The estimates of $\boldsymbol{h}$ are then used to build a polynomial whose derivatives at a regressor give an estimate of the parameters of the ARX model generating that regressor.

Paper contributions. In this paper, we show that when the number of models and the model orders are unknown and possibly different, the hybrid model parameters are no longer uniquely defined, but rather live on a manifold $\mathcal{H}$ of possible solutions. Although in principle this means that the recursive algorithm may not converge, we demonstrate that under suitable persistence of excitation conditions, the estimates of $\boldsymbol{h}$ do converge to a point in $\mathcal{H}$ that depends continuously on the initial condition. Given two such estimates obtained from two different initial conditions, we compute two polynomials whose derivatives at a regressor give an estimate of the parameters of the ARX model generating that regressor. After properly enforcing some of the entries of $\boldsymbol{h}$ to be zero, we obtain a recursive algorithm which, under suitable persistence of excitation conditions, gives an exponentially convergent estimate of the model parameters of each ARX model, without knowing the number of ARX models, the orders of the ARX models or the switching sequence.

Related literature. Most existing hybrid system identification methods have been designed for the class of piecewise affine/ARX systems, i.e. models in which the regressor space is partitioned into polyhedra with affine/ARX submodels for each polyhedron. [4] combines clustering, regression and classification techniques. [3] solves for the model parameters and the partition of the state space using mixed-integer linear and quadratic programming. [2] uses a greedy approach for partitioning a set of infeasible inequalities into a minimum number of feasible subsystems. [6] iterates between assigning data points to models and computing the model parameters using a Bayesian approach. For the class of SARX models with known and equal orders, [10], [8], [7] show that the identification problem can be solved in closed form by exploiting the properties of the so-called hybrid decoupling polynomial, an equation on the input/output data 
that does not depend on the switching sequence.

Unfortunately, most hybrid system identification algorithms are batch, i.e. the model parameters and the switching sequence are identified after all the input/output data have been collected. This is a significant limitation, because the computational complexity of batch algorithms depends on the number of data points, hence they may not be suitable for real time applications. To the best of our knowledge, other than [9], there is no prior work addressing the recursive identification of hybrid dynamical models in the case in which the number of models, the model orders, the model parameters, and the switching sequence are all unknown.

\section{RECURSIVE IDENTIFICATION OF ARX SYSTEMS}

Consider an ARX system with known orders $n_{a}$ and $n_{c}$

$y_{t}=a_{1} y_{t-1}+\cdots+a_{n_{a}} y_{t-n_{a}}+c_{1} u_{t-1}+\cdots+c_{n_{c}} u_{t-n_{c}}$.

If we let $K=n_{a}+n_{c}+1$,

$$
\begin{aligned}
\theta & \doteq\left[c_{n_{c}}, \ldots, c_{1}, a_{n_{a}}, \ldots, a_{1}\right]^{\top} \in \mathbb{R}^{K-1}, \text { and } \\
\psi_{t} & \doteq\left[u_{t-n_{c}}, \ldots, u_{t-1}, y_{t-n_{a}}, \ldots y_{t-1}\right]^{\top} \in \mathbb{R}^{K-1},
\end{aligned}
$$

then we have that for all $t \geq \max \left\{n_{a}, n_{c}\right\}$ the vector of regressors $\boldsymbol{x}_{t}$ lives in the following hyperplane of $\mathbb{R}^{K}$

$$
\boldsymbol{b}^{\top} \boldsymbol{x}_{t}=\left[\begin{array}{ll}
\theta^{\top} & 1
\end{array}\right]\left[\begin{array}{c}
\psi_{t} \\
-y_{t}
\end{array}\right]=0,
$$

whose normal vector is the vector of model parameters $\boldsymbol{b}$.

It is well known (see e.g. [1]) that if the ARX model is minimal ${ }^{1}$ and the input/output data are persistently exciting, i.e. there is an $S \in \mathbb{N}$ and $\rho_{1}, \rho_{2}>0$ such that for all $j^{2}$

$$
\rho_{1} I_{K-1} \prec \sum_{t=j}^{j+S} \psi_{t} \psi_{t}^{\top} \prec \rho_{2} I_{K-1},
$$

then the recursive equation error identifier

$$
\hat{\boldsymbol{b}}_{t+1}=\left(I_{K}-\frac{\mu \Pi_{1} \boldsymbol{x}_{t} \boldsymbol{x}_{t}^{\top}}{1+\mu\left\|\Pi_{1} \boldsymbol{x}_{t}\right\|^{2}}\right) \hat{\boldsymbol{b}}_{t},
$$

where $\Pi_{1}=\left[\begin{array}{cc}I_{K-1} & \mathbf{0}_{K-1} \\ \mathbf{0}_{K-1}^{\top} & 0\end{array}\right] \in \mathbb{R}^{K \times K}$ and $\mu>0$, produces an exponentially convergent estimate of the model parameters $\boldsymbol{b} \in \mathbb{R}^{K}$. That is, if $e_{t}=\hat{\boldsymbol{b}}_{t}-\boldsymbol{b}$, then

$$
\prod_{i=0}^{T} F_{i}=\prod_{i=0}^{t}\left(I_{K}-\frac{\mu \Pi_{1} \boldsymbol{x}_{i} \boldsymbol{x}_{i}^{\top} \Pi_{1}}{1+\mu\left\|\Pi_{1} \boldsymbol{x}_{i}\right\|^{2}}\right) \rightarrow \mathbf{0}_{K \times K}
$$

exponentially, so that for all $e_{0} \in \mathbb{R}^{K}, e_{t} \rightarrow \mathbf{0}_{K}$, because

$$
e_{t+1}=\left(I_{K}-\frac{\mu \Pi_{1} \boldsymbol{x}_{t} \boldsymbol{x}_{t}^{\top} \Pi_{1}}{1+\mu\left\|\Pi_{1} \boldsymbol{x}_{t}\right\|^{2}}\right) e_{t}=F_{t} e_{t}=\prod_{i=0}^{t} F_{i} e_{0} .
$$

In practice, the model orders may be unknown, and only upper bounds $\bar{n}_{a}$ and $\bar{n}_{c}$ may be available. In this case, the model parameters and regressors are given by

$$
\begin{aligned}
\boldsymbol{b} & \doteq\left[\mathbf{0}_{\bar{n}_{c}-n_{c}}, c_{n_{c}}, \ldots, c_{1}, \mathbf{0}_{\bar{n}_{a}-n_{a}}, a_{n_{a}}, \ldots, a_{1}, 1\right]^{\top}, \\
\boldsymbol{x}_{t} & \doteq\left[u_{t-\bar{n}_{c}}, \ldots, u_{t-1}, y_{t-\bar{n}_{a}}, \ldots, y_{t-1},-y_{t}\right]^{\top} \in \mathbb{R}^{K},
\end{aligned}
$$

\footnotetext{
${ }^{1}$ The numerator and denominator of the transfer function are coprime. ${ }^{2} A \prec B$ means that $(B-A)$ is positive definite.
}

where $K=\bar{n}_{a}+\bar{n}_{c}+1$. Notice that here the vector $\boldsymbol{b}$ is the same as one in (2) with additional $\bar{n}_{a}-n_{a}$ and $\bar{n}_{c}-n_{c}$ zeros filled in before the terms $a_{n_{a}}$ and $c_{n_{c}}$, respectively. Notice also that due to the redundant embedding (10), the vector $\boldsymbol{b}$ is no longer the only vector orthogonal to all the regressors. It is easy to verify that the following $\min \left(\bar{n}_{a}-n_{a}, \bar{n}_{c}-n_{c}\right)$ vectors are also orthogonal to $\boldsymbol{x}_{t}$ for all $t \geq 0$

$$
\begin{aligned}
\boldsymbol{b}^{1} & =\left[\mathbf{0}_{\bar{n}_{c}-n_{c}-1}, c_{n_{c}}, \ldots, c_{1}, \mathbf{0}_{1}, \mathbf{0}_{\bar{n}_{a}-n_{a}-1}, a_{n_{a}}, \ldots, a_{1},-1, \mathbf{0}_{1}\right]^{\top} \\
\boldsymbol{b}^{2} & =\left[\mathbf{0}_{\bar{n}_{c}-n_{c}-2}, c_{n_{c}}, \ldots, c_{1}, \mathbf{0}_{2}, \mathbf{0}_{\bar{n}_{a}-n_{a}-2}, a_{n_{a}}, \ldots, a_{1},-1, \mathbf{0}_{2}\right]^{\top}
\end{aligned}
$$

Therefore, if $\bar{n}_{a}>n_{a}$ and $\bar{n}_{c}>n_{c}$ the regressor vectors $\left\{\boldsymbol{x}_{t}\right\}$ are no longer persistently exciting, because they span a linear subspace $\mathcal{S} \subset \mathbb{R}^{K}$ of dimension $d=\bar{n}_{a}+\bar{n}_{c}-$ $\min \left(\bar{n}_{a}-n_{a}, \bar{n}_{c}-n_{c}\right)<K-1$, thus violating the left hand side of (5). As a consequence, the identifier (6) is no longer guaranteed to converge to the true parameter vector $\boldsymbol{b}$.

We now show that although the regressors are not persistently exciting, $\hat{\boldsymbol{b}}_{t}$ still converges to a point in the subspace $\mathcal{S}^{\perp}=\operatorname{span}\left\{\boldsymbol{b}, \boldsymbol{b}^{1}, \boldsymbol{b}^{2}, \cdots\right\}$ which depends linearly on the initial condition $\hat{\boldsymbol{b}}_{0}$. To see this, assume that the regressors span a $d$-dimensional subspace. Then there exists an orthogonal matrix $R \in S O(K-1)$ such that $\psi_{t}=R\left[\begin{array}{c}z_{t} \\ \mathbf{0}_{K-d-1}\end{array}\right]$ for some $\boldsymbol{z}_{t} \in \mathbb{R}^{d}$. Therefore, from (8) we have

$$
\begin{aligned}
F_{t} & =\left[\begin{array}{cc}
R & \mathbf{0}_{K-1} \\
\mathbf{0}_{K-1}^{\top} & 1
\end{array}\right]\left(I_{K}-\frac{\mu\left[\begin{array}{c}
z_{t} \\
\mathbf{0}_{K-d}
\end{array}\right]\left[\begin{array}{c}
z_{t} \\
\mathbf{0}_{K-d}
\end{array}\right]}{1+\mu\left\|z_{t}\right\|^{2}}\right)\left[\begin{array}{cc}
R^{\top} & \mathbf{0}_{K-1} \\
\mathbf{0}_{K-1}^{\top} & 1
\end{array}\right] \\
& =\left[\begin{array}{cc}
R & \mathbf{0}_{K-1}^{\top} \\
\mathbf{0}_{K-1}^{\top} & 1
\end{array}\right]\left[\begin{array}{cc}
I_{d}-\frac{\mu z_{t} z_{t}^{\top}}{1+\mu\left\|z_{t}\right\|^{2}} & \mathbf{0}_{d \times(K-d)} \\
\mathbf{0}_{(K-d) \times d} & I_{K-d}
\end{array}\right]\left[\begin{array}{cc}
R^{\top} & \mathbf{0}_{K-1} \\
\mathbf{0}_{K-1}^{\top} & 1
\end{array}\right]
\end{aligned}
$$

hence

$\prod_{i=0}^{t} F_{i} \rightarrow\left[\begin{array}{cc}R & \mathbf{0}_{K-1} \\ \mathbf{0}_{K-1}^{\top} & 1\end{array}\right]\left[\begin{array}{cc}\mathbf{0}_{d \times d} & \mathbf{0}_{d \times(K-d)} \\ \mathbf{0}_{(K-d) \times d} & I_{K-d}\end{array}\right]\left[\begin{array}{cc}R^{\top} & \mathbf{0}_{K-1} \\ \mathbf{0}_{K-1}^{\top} & 1\end{array}\right]$,

where the last step follows from (7) under the assumption that the vectors $z_{t}$ are persistently exciting. Therefore, if we let $R=\left[\begin{array}{ll}R_{1} & R_{2}\end{array}\right]$, the error $e_{t}=\prod_{i=0}^{t-1} F_{i} e_{0}$ converges to

$$
e^{*}=\boldsymbol{b}^{*}-\boldsymbol{b}=R_{2} R_{2}^{\top} e_{0}=R_{2} R_{2}^{\top}\left(\hat{\boldsymbol{b}}_{0}-\boldsymbol{b}\right),
$$

where $R_{2} \in \mathbb{R}^{(K-1) \times(K-d-1)}$ are the last $K-d-1$ columns of $R$. The vector of parameters converges to

$$
\boldsymbol{b}^{*}=\boldsymbol{b}+R_{2} R_{2}^{\top}\left(\hat{\boldsymbol{b}_{0}}-\boldsymbol{b}\right) .
$$

We have shown the following result.

Theorem 1: If the regressors formed with orders $\bar{n}_{a} \geq$ $n_{a}$ and $\bar{n}_{c} \geq n_{c}$ form a subspace of dimension $d=$ $\bar{n}_{a}+\bar{n}_{c}-\min \left\{\bar{n}_{a}-n_{a}, \bar{n}_{c}-n_{c}\right\}$, and their projections onto this $d$-dimensional space are persistently exciting, then the recursive error identifier $\hat{\boldsymbol{b}}_{t}$ converges exponentially to a point in the orthogonal complement to the span of the regressors that depends linearly on the initial condition. If in addition $\bar{n}_{a}=n_{a}$ or $\bar{n}_{c}=n_{c}$, then $\hat{\boldsymbol{b}}_{t} \rightarrow \boldsymbol{b}$ exponentially. 
The important point to notice is that Theorem 1 guarantees convergence to a point for any $\bar{n}_{a} \geq n_{a}$ and $\bar{n}_{c} \geq n_{c}$. Therefore, we may run two identifiers in parallel with different initial conditions. If the two identifiers converge to different parameter vectors, then we know that $\bar{n}_{a}$ and $\bar{n}_{c}$ are not strict, hence we may reduce these upper bounds by one until the two identifiers converge to the same parameter vector $\boldsymbol{b}$. Once $\boldsymbol{b}$ has been identified, $n_{a}$ and $n_{c}$ can be obtained from the number and location of its zero entries.

\section{RECURSIVE IDENTIFICATION OF SARX Systems}

This section presents a solution to Problem 1 under the following assumption of minimality of the SARX model:

Definition 1: An SARX model is said to be minimal if

1) For all $i=1, \ldots, n$, the $i$ th ARX model is minimal.

2) For all $i \neq j=1, \ldots, n$ the transfer functions $H_{i}(z)$ and $H_{j}(z)$ are such that $H_{i}(z) \neq H_{j}(z)$ for all $z \in \mathbb{C}$.

\section{A. The hybrid model parameters}

Let $\bar{n}_{a} \geq \max \left\{n_{a}(i)\right\}$ and $\bar{n}_{c} \geq \max \left\{n_{c}(i)\right\}$ be given upper bounds on the orders of the ARX models. Notice from (1) that for all $t \geq \max \left\{\bar{n}_{a}, \bar{n}_{c}\right\}$ there exists a discrete state $\lambda_{t}=i \in\{1, \ldots, n\}$ such that $\boldsymbol{b}_{i}^{\top} \boldsymbol{x}_{t}=0$, where

$\boldsymbol{b}_{i} \doteq\left[\mathbf{0}_{\bar{n}_{c}-n_{c}}^{\top}, c_{n_{c}}(i), \ldots, c_{1}(i), \mathbf{0}_{\bar{n}_{a}-n_{a}}^{\top}, a_{n_{a}}(i), \ldots, a_{1}(i), 1\right]^{\top}$.

Therefore, the following hybrid decoupling polynomial must be satisfied by the model parameters and the input/output data for any possible value of the discrete state

$$
\prod_{i=1}^{n}\left(\boldsymbol{b}_{i}^{\top} \boldsymbol{x}_{t}\right)=0 \text {. }
$$

The hybrid decoupling polynomial is simply a homogeneous polynomial of degree $n$ in $K$ variables

$$
p_{n}(\boldsymbol{z}) \doteq \prod_{i=1}^{n}\left(\boldsymbol{b}_{i}^{\top} \boldsymbol{z}\right)=\boldsymbol{h}^{\top} \nu_{n}(\boldsymbol{z})=0,
$$

that can be written as a linear combination of the $M_{n}(K)=$ $\left(\begin{array}{c}n+K-1 \\ K-1\end{array}\right)=\left(\begin{array}{c}n+K-1 \\ n\end{array}\right)$ monomials $z_{1}^{n_{1}} z_{2}^{n_{2}} \cdots z_{K}^{n_{K}}$ with $0 \leq$ $n_{j} \leq n$ for $j=1, \ldots, K$, and $n_{1}+n_{2}+\cdots+n_{K}=n$. In (15) $\nu_{n}: \mathbb{R}^{K} \rightarrow \mathbb{R}^{M_{n}(K)}$ is the stack of all such monomials:

$$
\nu_{n}:\left[z_{1}, \ldots, z_{K}\right]^{\top} \mapsto\left[\ldots, \boldsymbol{z}_{1}^{n_{1}} \ldots z_{K}^{n_{K}}, \ldots\right]^{\top},
$$

with $n_{1}, \ldots, n_{K}$ chosen in the degree-lexicographic order. $\nu_{n}$ is also known as Veronese map of degree $n$ [5]. The coefficients are written as a vector $\boldsymbol{h} \in \mathbb{R}^{M_{n}(K)}$ and are referred to as the hybrid model parameters.

\section{B. Recursive identification of the hybrid model parameters}

We now show how to generalize the recursive identifier in (6) and its convergence properties to the case of SARX models such as (1). It was shown in [9] that thanks to the hybrid decoupling polynomial, one can derive a recursive identification algorithm that operates on the hybrid model parameters $\boldsymbol{h}$ rather than on the ARX model parameters $\left\{\boldsymbol{b}_{i}\right\}_{i=1}^{n}$. Given an estimate for $\boldsymbol{h}$, one can easily identify the ARX model parameters $\left\{\boldsymbol{b}_{i}\right\}_{i=1}^{n}$, as we will show later.
The advantage of identifying $\boldsymbol{h}$ first is that the hybrid model parameters do not depend on the value of the discrete state or the switching mechanism. Therefore, one can use a standard recursive identifier to identify $\boldsymbol{h}$, as we show now.

In order to derive a recursive identifier for SARX models, let $\Pi_{n}=\left[\begin{array}{cc}I_{M_{n}(K)-1} & \mathbf{0}_{M_{n}(K)-1} \\ \mathbf{0}_{M_{n}(K)-1}^{\top} & 0\end{array}\right] \in \mathbb{R}^{M_{n}(K) \times M_{n}(K)}$. Then the equation $\boldsymbol{h}^{\top} \nu_{n}\left(\boldsymbol{x}_{t}\right)=0$ defines an ARX model in a lifted space with output

$$
z_{t}=(-1)^{n} y_{t}^{n}=\left(\Pi_{n} \boldsymbol{h}\right)^{\top}\left(\Pi_{n} \nu_{n}\left(\boldsymbol{x}_{t}\right)\right)=\sum_{j=0}^{M_{n}(K)-2} h_{j} v_{t-j}
$$

and input $\left[v_{t-1}, \ldots, v_{t-M_{n}(K)+1}\right]^{\top}=\Pi_{n} \nu_{n}\left(\boldsymbol{x}_{t}\right)$. After applying the results of Section II to the model (17), we obtain the following hybrid equation error identifier for SARX systems [9]:

$$
\hat{\boldsymbol{h}}_{t+1}=\left(I_{M_{n}(K)}-\mu \frac{\Pi_{n} \nu_{n}\left(\boldsymbol{x}_{t}\right) \nu_{n}\left(\boldsymbol{x}_{t}\right)^{\top}}{1+\mu\left\|\Pi_{n} \nu_{n}\left(\boldsymbol{x}_{t}\right)\right\|^{2}}\right) \hat{\boldsymbol{h}}_{t} .
$$

In the following subsections we characterize the exponential convergence of the hybrid equation error identifier (18) in terms of the regressors $\left\{\boldsymbol{x}_{t}\right\}$ generated by the SARX model. For ease of exposition, we consider the following three cases of increasing complexity: 1) known number of models and known and equal orders; 2) unknown number of models and known and equal orders; 3) unknown number of models and unknown and possibly different orders.

1) Known number of models and known and equal orders: The case in which the number of models $n$ is known and the model orders are known and equal, i.e. $n_{a}=n_{a}(1)=\cdots=$ $n_{a}(n)$ and $n_{c}=n_{c}(1)=\cdots=n_{c}(n)$, has been studied in our previous work [9]. We have shown that in this case both the hybrid model parameters and the ARX model parameters converge exponentially to their true values as stated in the following theorem.

Theorem 2: Consider a minimal SARX system of the form (1) and assume that the following recursive identification scheme is used

$$
\begin{aligned}
\hat{\boldsymbol{h}}_{t+1} & =\left(I_{M_{n}(K)}-\mu \frac{\Pi_{n} \nu_{n}\left(\boldsymbol{x}_{t}\right) \nu_{n}\left(\boldsymbol{x}_{t}\right)^{\top}}{1+\mu\left\|\Pi_{n} \nu_{n}\left(\boldsymbol{x}_{t}\right)\right\|^{2}}\right) \hat{\boldsymbol{h}}_{t} \\
\hat{\boldsymbol{b}}_{t} & =\frac{D \nu_{n}^{\top}\left(\boldsymbol{x}_{t}\right) \hat{\boldsymbol{h}}_{t}}{e_{K}^{\top} D \nu_{n}^{\top}\left(\boldsymbol{x}_{t}\right) \hat{\boldsymbol{h}}_{t}}
\end{aligned}
$$

where $e_{K}=[0, \cdots, 0,1]^{\top} \in \mathbb{R}^{K}$ and $D \nu_{n}\left(\boldsymbol{x}_{t}\right)$ is the Jacobian of $\nu_{n}$ at $\boldsymbol{x}_{t}$. If there exist $\rho_{1}, \rho_{2}>0$ and an integer $S$ such that for all $j \geq \max \left\{n_{a}, n_{c}\right\}$

$$
\rho_{1} I_{M_{n}(K)-1} \prec \sum_{t=j}^{j+S} \Pi_{n} \nu_{n}\left(\boldsymbol{x}_{t}\right) \nu_{n}^{\top}\left(\boldsymbol{x}_{t}\right) \Pi_{n}^{\top} \prec \rho_{2} I_{M_{n}(K)-1},
$$

then $\widehat{\boldsymbol{h}}_{t}-\boldsymbol{h} \rightarrow \mathbf{0}$ and $\hat{\boldsymbol{b}}_{t}-\boldsymbol{b}_{\lambda_{t}} \rightarrow \mathbf{0}$ exponentially. Furthermore,

$$
\boldsymbol{b}_{\lambda_{t}}=\frac{D \nu_{n}^{\top}\left(\boldsymbol{x}_{t}\right) \boldsymbol{h}}{e_{K}^{\top} D \nu_{n}^{\top}\left(\boldsymbol{x}_{t}\right) \boldsymbol{h}}
$$


2) Unknown number of models and known and equal orders: In practice the number of models $n$ may be unknown, and only an upper bound $\bar{n} \geq n$ may be available. In this case, the embedded regressors $\nu_{\bar{n}}\left(\boldsymbol{x}_{t}\right)$ are no longer persistently exciting in the sense of (21), because they live in a linear subspace of $\mathbb{R}^{M_{\bar{n}}}$ of dimension $M_{\bar{n}}(K)-M_{\bar{n}-n}(K)$ which is strictly less than $M_{\bar{n}}(K)-1$ if $\bar{n}>n$, as stated by the following lemma.

Lemma 1: Let $\left\{S_{i} \subset \mathbb{R}^{K}\right\}_{i=1}^{n}$ be a collection of $n$ linear subspaces of $\mathbb{R}^{K}$ of dimension $K-1$. The dimension of the span of $\nu_{\bar{n}}\left(\cup_{i=1}^{n} S_{i}\right)$ is $M_{\bar{n}}(K)-M_{\bar{n}-n}(K)$ for $\bar{n} \geq n$.

Proof. Let $p_{\bar{n}}(\boldsymbol{x})=\boldsymbol{c}^{\top} \nu_{\bar{n}}(\boldsymbol{x})$ be any homogeneous polynomial of degree $\bar{n}$ that vanishes on $\nu_{\bar{n}}\left(\cup_{i=1}^{n} S_{i}\right)$. Then, $p_{\bar{n}}$ must have $p_{n}(\boldsymbol{x})=\left(\boldsymbol{b}_{1}^{\top} \boldsymbol{x}\right) \cdots\left(\boldsymbol{b}_{n}^{\top} \boldsymbol{x}\right)$ as a factor, i.e. $p_{\bar{n}}=p_{n} q_{\bar{n}-n}$, where $q_{\bar{n}-n}$ is any polynomial of degree $\bar{n}-n$. There are $M_{\bar{n}-n}(K)$ such linearly independent polynomials, and so there are $M_{\bar{n}-n}(K)$ vectors $c$ in the orthogonal complement to the span of $\nu_{\bar{n}}\left(\cup_{i=1}^{n} S_{i}\right)$. Therefore, the dimension of the span of $\nu_{\bar{n}}\left(\cup_{i=1}^{n} S_{i}\right)$ is $M_{\bar{n}}(K)-$ $M_{\bar{n}-n}(K)$ for $\bar{n} \geq n$ as claimed.

Fortunately, thanks to Theorem 1 we know that even though the vectors $\nu_{n}\left(\boldsymbol{x}_{t}\right)$ may not be persistently exciting, the hybrid recursive identifier still converges to a point $\boldsymbol{h}^{*}$ in the orthogonal complement to the span of $\left\{\nu_{n}\left(\boldsymbol{x}_{t}\right)\right\}$, i.e. $\boldsymbol{h}^{*}$ is such that $p_{\bar{n}}\left(\boldsymbol{x}_{t}\right)=\nu_{\bar{n}}\left(\boldsymbol{x}_{t}\right)^{\top} \boldsymbol{h}^{*}=0$ for all $t \geq 0$. However, we do not yet know what polynomial the vector $\boldsymbol{h}^{*}$ corresponds to, and hence we do not know whether the derivatives of $p_{\bar{n}}$ would still converge to the true $\left\{\boldsymbol{b}_{i}\right\}_{i=1}^{n}$. The following theorem shows that $\hat{\boldsymbol{b}}_{t}-\boldsymbol{b}_{\lambda_{t}}$ still converges to $\mathbf{0}_{K}$ exponentially for almost all initial conditions $\boldsymbol{h}_{0}$.

Theorem 3: Consider a minimal SARX system of the form (1) and assume that the following recursive identification scheme with $\bar{n}>n$ modes is used

$$
\begin{aligned}
\hat{\boldsymbol{h}}_{t+1} & =\left(I_{M_{\bar{n}}(K)}-\mu \frac{\Pi_{\bar{n}} \nu_{\bar{n}}\left(\boldsymbol{x}_{t}\right) \nu_{\bar{n}}\left(\boldsymbol{x}_{t}\right)^{\top}}{1+\mu\left\|\Pi_{\bar{n}} \nu_{\bar{n}}\left(\boldsymbol{x}_{t}\right)\right\|^{2}}\right) \hat{\boldsymbol{h}}_{t} \\
\hat{\boldsymbol{b}}_{t} & =\frac{D \nu_{\bar{n}}^{\top}\left(\boldsymbol{x}_{t}\right) \hat{\boldsymbol{h}}_{t}}{e_{K}^{\top} D \nu_{\bar{n}}^{\top}\left(\boldsymbol{x}_{t}\right) \hat{\boldsymbol{h}}_{t}} .
\end{aligned}
$$

If there exist $\rho_{1}, \rho_{2}>0$ and an integer $S$ such that for all $j \geq \max \left\{n_{a}, n_{c}\right\}$

$$
\rho_{1} I_{d} \prec \sum_{t=j}^{j+S} \Gamma_{d} \nu_{\bar{n}}\left(\boldsymbol{x}_{t}\right) \nu_{\bar{n}}^{\top}\left(\boldsymbol{x}_{t}\right) \Gamma_{d}^{\top} \prec \rho_{2} I_{d},
$$

where $d=M_{\bar{n}}(K)-M_{\bar{n}-n}(K)$ and $\Gamma_{d} \in \mathbb{R}^{d \times M_{\bar{n}}(K)}$ is a projection matrix onto the subspace spanned by the embedded regressors, then $\hat{\boldsymbol{b}}_{t}-\boldsymbol{b}_{\lambda_{t}} \rightarrow \mathbf{0}$ exponentially for an open and dense set of initial conditions $\boldsymbol{h}_{0}$.

Proof. Let $\boldsymbol{h}^{*}$ be the vector of hybrid model parameters to which the hybrid recursive identifier converges. We know that the polynomial $p_{\bar{n}}(\boldsymbol{z})=\nu_{\bar{n}}(\boldsymbol{z})^{\top} \boldsymbol{h}^{*}$ is such that $p_{\bar{n}}\left(\boldsymbol{x}_{t}\right)=0$ for all $t \geq 0$. In addition, we know that the vectors $\Gamma_{d} \nu_{n}\left(\boldsymbol{x}_{t}\right)$ are persistently exciting. Therefore, since the vectors $\boldsymbol{x}_{t}$ live in a union of $n$ hyperplanes, the polynomial $p_{\bar{n}}(\boldsymbol{z})$ must vanish in the entire set $\cup_{i=1}^{n} Z_{i}$, where $Z_{i}$ is the set of all points $z$ such $\boldsymbol{b}_{i}^{\top} \boldsymbol{z}=0$. Note also that since the SARX system is minimal, the vectors $\boldsymbol{b}_{1}, \ldots, \boldsymbol{b}_{n}$ are different, hence any polynomial that vanishes on the set $\cup_{i=1}^{n} Z_{i}$ must have $p_{n}(\boldsymbol{z})=\left(\boldsymbol{b}_{1}^{\top} \boldsymbol{z}\right) \cdots\left(\boldsymbol{b}_{n}^{\top} \boldsymbol{z}\right)$ as a factor. Therefore, the polynomial $p_{\bar{n}}$ must be of the form

$$
p_{\bar{n}}(\boldsymbol{z})=p_{n}(\boldsymbol{z}) q_{\bar{n}-n}(\boldsymbol{z})
$$

for some polynomial $q_{\bar{n}-n}(\boldsymbol{z})$ of degree $\bar{n}-n$. Therefore, the derivative of $p_{\bar{n}}$ is

$$
D p_{\bar{n}}(\boldsymbol{z})=D p_{n}(\boldsymbol{z}) q_{\bar{n}-n}(\boldsymbol{z})+p_{n}(\boldsymbol{z}) D q_{\bar{n}-n}(\boldsymbol{z}) .
$$

If $\boldsymbol{z}=\boldsymbol{x}_{t}$, then $p_{n}\left(\boldsymbol{x}_{t}\right)=0$, hence $D p_{\bar{n}}\left(\boldsymbol{x}_{t}\right) \sim D p_{n}\left(\boldsymbol{x}_{t}\right)$ whenever $q_{\bar{n}-n}\left(\boldsymbol{x}_{t}\right) \neq 0$. The latter follows from the fact that for an open and dense set of initial conditions the polynomial $q_{\bar{n}-n}(\boldsymbol{z})$ has no common factor with $p_{n}$. Combining (27) with (22) we have

$$
\boldsymbol{b}_{\lambda_{t}}=\frac{D p_{n}\left(\boldsymbol{x}_{t}\right)}{e_{K}^{\top} D p_{n}\left(\boldsymbol{x}_{t}\right)}=\frac{D \nu_{\bar{n}}^{\top}\left(\boldsymbol{x}_{t}\right) \boldsymbol{h}^{*}}{e_{K}^{\top} D \nu_{\bar{n}}^{\top}\left(\boldsymbol{x}_{t}\right) \boldsymbol{h}^{*}} .
$$

Now, from the exponential convergence of $\hat{\boldsymbol{h}}_{t}$ there exist $\kappa, \lambda>$ 0 such that $\left\|\hat{\boldsymbol{h}}_{t}-\boldsymbol{h}^{*}\right\|<\kappa \lambda^{-t}$. In addition, the vectors $\boldsymbol{b}_{1}, \ldots, \boldsymbol{b}_{n}$ are different, because the SARX model is minimal, hence the polynomial $\boldsymbol{h}^{* T} \nu_{\bar{n}}(\boldsymbol{z})$ has no repeated factor. Therefore, there is a $\delta>0$ and a $T>0$ such that for all $t>T$ we have $\left\|D \nu_{\bar{n}}\left(\boldsymbol{x}_{t}\right)^{\top} \boldsymbol{h}^{*}\right\| \geq \delta$ and $\left\|D \nu_{\bar{n}}\left(\boldsymbol{x}_{t}\right)^{\top} \hat{\boldsymbol{h}}_{t}\right\| \geq \delta$ (see proof of Theorem 3 in [9] for the latter claim). In addition, note that $\left\|\nu_{\bar{n}}\left(\boldsymbol{x}_{t}\right)\right\|^{2}=\left\|\Gamma_{d} \nu_{\bar{n}}\left(\boldsymbol{x}_{t}\right)\right\|^{2}+$ $\sum_{i=1}^{d}\left(e_{M_{\bar{n}}(K)-i}^{\top} \nu_{\bar{n}}\left(\boldsymbol{x}_{t}\right)\right)^{2}=\left\|\Gamma_{d} \nu_{\bar{n}}\left(\boldsymbol{x}_{t}\right)\right\|^{2}\left(1+\sum_{i=1}^{d}\left\|\boldsymbol{g}_{i}\right\|^{2}\right)$, because the last $d$ entries of $\nu_{\bar{n}}\left(\boldsymbol{x}_{t}\right)$ are linear combinations of $\Gamma_{d} \nu_{\bar{n}}\left(\boldsymbol{x}_{t}\right)$ with coefficients $\boldsymbol{g}_{i}, i=1, \ldots, d$. Combining this with (24) and (28) yields

$$
\begin{aligned}
& \left\|\boldsymbol{b}_{\lambda_{t}}-\hat{\boldsymbol{b}}_{\lambda_{t}}\right\|= \\
& \left\|\frac{e_{K}^{\top} D \nu_{\bar{n}}^{\top}\left(\boldsymbol{x}_{t}\right) \hat{\boldsymbol{h}}_{t} D \nu_{\bar{n}}^{\top}\left(\boldsymbol{x}_{t}\right) \boldsymbol{h}^{*}-e_{K}^{\top} D \nu_{\bar{n}}^{\top}\left(\boldsymbol{x}_{t}\right) \boldsymbol{h}^{*} D \nu_{\bar{n}}^{\top}\left(\boldsymbol{x}_{t}\right) \hat{\boldsymbol{h}}_{t}}{\left|e_{K}^{\top} D \nu_{\bar{n}}^{\top}\left(\boldsymbol{x}_{t}\right) \hat{\boldsymbol{h}}_{t} \| e_{K}^{\top} D \nu_{\bar{n}}^{\top}\left(\boldsymbol{x}_{t}\right) \boldsymbol{h}^{*}\right|}\right\| \\
& \left\|\frac{e_{K}^{\top}\left(D \nu_{\bar{n}}^{\top}\left(\boldsymbol{x}_{t}\right)\left(\hat{\boldsymbol{h}}_{t}-\boldsymbol{h}^{*}\right) D \nu_{\bar{n}}^{\top}\left(\boldsymbol{x}_{t}\right) \boldsymbol{h}^{*}-D \nu_{\bar{n}}^{\top}\left(\boldsymbol{x}_{t}\right) \boldsymbol{h}^{*} D \nu_{\bar{n}}^{\top}\left(\boldsymbol{x}_{t}\right)\left(\hat{\boldsymbol{h}}_{t}-\boldsymbol{h}^{*}\right)\right)}{\left|e_{K}^{\top} D \nu_{\bar{n}}^{\top}\left(\boldsymbol{x}_{t}\right) \hat{\boldsymbol{h}}_{t} \| e_{K}^{\top} D \nu_{\bar{n}}^{\top}\left(\boldsymbol{x}_{t}\right) \boldsymbol{h}^{*}\right|}\right\| \\
& \leq 2 \frac{\left\|D \nu_{\bar{n}}^{\top}\left(\boldsymbol{x}_{t}\right)\left(\hat{\boldsymbol{h}}_{t}-\boldsymbol{h}^{*}\right)\right\|\left\|D \nu_{\bar{n}}^{\top}\left(\boldsymbol{x}_{t}\right) \boldsymbol{h}^{*}\right\|}{\left|e_{K}^{\top} D \nu_{\bar{n}}^{\top}\left(\boldsymbol{x}_{t}\right) \hat{\boldsymbol{h}}_{t} \| e_{K}^{\top} D \nu_{\bar{n}}^{\top}\left(\boldsymbol{x}_{t}\right) \boldsymbol{h}^{*}\right|} \leq 2 \frac{\alpha_{\bar{n}}^{2} E_{\bar{n}}^{2}\left\|\boldsymbol{h}^{*}\right\| \kappa \lambda^{-t}}{\delta^{2}} .
\end{aligned}
$$

In the last step we have used the fact that for all $\boldsymbol{z} \in \mathbb{R}^{K}$ there is a constant matrix of exponents $E_{j \bar{n}} \in \mathbb{R}^{M_{\bar{n}}(K) \times M_{\bar{n}-1}(K)}$ such that $\partial \nu_{\bar{n}}(\boldsymbol{z}) / \partial z_{j}=E_{j \bar{n}} \nu_{\bar{n}-1}(\boldsymbol{z})$. Therefore, $\left\|D \nu_{\bar{n}}(\boldsymbol{z})\right\| \leq$ $E_{\bar{n}}\left\|\nu_{\bar{n}-1}(\boldsymbol{z})\right\|=E_{\bar{n}} \frac{\bar{n}}{\bar{n}-1} \sqrt{\left\|\nu_{\bar{n}}(\boldsymbol{z})\right\|} \leq \alpha_{\bar{n}} E_{\bar{n}}$, where $E_{\bar{n}}=$ $\max _{j=1, \ldots, K}\left(\left\|E_{j \bar{n}}\right\|\right)$ and $\alpha_{\bar{n}}=\frac{\bar{n}}{2(\bar{n}-1)} \sqrt{\rho_{2}\left(1+\sum_{i=1}^{d}\left\|\boldsymbol{g}_{i}\right\|^{2}\right)}$.

In summary, we have shown that even if the number of models is overestimated, under suitable persistence of excitation conditions the recursive identifier always converges to the coefficients of a polynomial that has $p_{n}(\boldsymbol{z})$ as a factor. Hence the derivatives of this polynomial always give an exponentially convergent estimate of the parameter vectors of each one of the ARX models.

Notice also that the theorem allows us to identify the number of modes. We can run two recursive identifiers $\hat{\boldsymbol{h}}_{t}^{1}$ and $\hat{\boldsymbol{h}}_{t}^{2}$ starting from two different initial conditions. If the two identifiers converge to different parameter vectors, but their corresponding estimates of the ARX model parameters are such that $\hat{\boldsymbol{b}}_{t}^{1}-\hat{\boldsymbol{b}}_{t}^{2} \rightarrow \mathbf{0}$, then we know that the number of models has been overestimated. Therefore, we may reduce the upper bound on the number of models by one until the two identifiers converge to the same solution, which only happens when $\bar{n}=n$. 
3) Unknown number of models and unknown and possibly different orders: Let us now consider the most challenging case in which both the number of models and the model orders are unknown, but we are given upper bounds $\bar{n} \geq n$, $\bar{n}_{a} \geq \max \left\{n_{a}(i)\right\}$ and $\bar{n}_{c} \geq \max \left\{n_{c}(i)\right\}$.

From our analysis in Section II, we know that the hybrid recursive identifier will converge to a point $\boldsymbol{h}^{*}$ in the orthogonal complement of the embedded regressors $\nu_{\bar{n}}\left(\boldsymbol{x}_{t}\right)$. Furthermore, it follows from the proof of Theorem 3 that if the orders are known and equal, then the polynomial identified by the recursive algorithm $p_{\bar{n}}(\boldsymbol{z})=\nu_{\bar{n}}(\boldsymbol{z})^{\top} \boldsymbol{h}^{*}$ always contains $p_{n}(\boldsymbol{z})=\left(\boldsymbol{b}_{1}^{\top} \boldsymbol{z}\right) \cdots\left(\boldsymbol{b}_{n}^{\top} \boldsymbol{z}\right)$ as a factor. Therefore, $p_{\bar{n}}$ produces the same derivatives (up to scale) as those of $p_{n}$, thus correctly identifying the parameter vectors $\boldsymbol{b}_{1}, \cdots, \boldsymbol{b}_{n}$. When the orders are unknown and possibly different, by following the same arguments as in the proof of Theorem 3 , the polynomial $p_{\bar{n}}$ must still factor as a product of two polynomials $p_{n}$ and $p_{\bar{n}-n}$ of degrees $n$ and $\bar{n}-n$, where $p_{n}\left(\boldsymbol{x}_{t}\right)=0$ for all $t \geq 0$. However, because the orders are unknown and possibly different, the polynomial $\left(\boldsymbol{b}_{1}^{\top} \boldsymbol{z}\right) \cdots\left(\boldsymbol{b}_{n}^{\top} \boldsymbol{z}\right)$ is no longer the only polynomial that vanishes on the entire set of regressors $\left\{\boldsymbol{x}_{t}\right\} .{ }^{3}$ Therefore $p_{\bar{n}}$ is not guaranteed to have $\left(\boldsymbol{b}_{1}^{\top} \boldsymbol{z}\right) \cdots\left(\boldsymbol{b}_{n}^{\top} \boldsymbol{z}\right)$ as a factor.

We show now that it is still possible to recover a polynomial in the orthogonal complement of $\left\{\nu_{\bar{n}}\left(\boldsymbol{x}_{t}\right)\right\}$ that has $\left(\boldsymbol{b}_{1}^{\top} \boldsymbol{z}\right) \cdots\left(\boldsymbol{b}_{n}^{\top} \boldsymbol{z}\right)$ as a factor by restricting some of the entries of $\hat{\boldsymbol{h}}_{t}$ to be zero. When two ARX models are of different orders, one of the models must be such that one or more of the leading entries of its vector of model parameters $\boldsymbol{b}$ are zero. Therefore, one or more of the leading entries of $\boldsymbol{h}$ must be zero. More generally, one can show that (see [7] for the proof)

1) The embedded regressors $\left\{\nu_{\bar{n}}\left(\boldsymbol{x}_{t}\right)\right\}$ live in a subspace of dimension $M_{\bar{n}}(K)-d-1$, where $d$ depends in a nontrivial way on $\bar{n}, \bar{n}_{a}, \bar{n}_{c},\left\{n_{a}(i)\right\},\left\{n_{c}(i)\right\}$.

2) The last $M_{\bar{n}}(K)-d$ entries of the embedded regressors, i.e. $\left\{\Gamma_{d} \nu_{\bar{n}}\left(\boldsymbol{x}_{t}\right)\right\}$ where $\Gamma_{d}=$ $\left[\mathbf{0}_{\left(M_{\bar{n}}(K)-d\right) \times d} I_{M_{\bar{n}}(K)-d}\right.$, live in a subspace of dimension $M_{\bar{n}}(K)-d-1$.

As a consequence of these two statements, we know that there exists a $d \geq 0$ such that the vectors $\left\{\Gamma_{d} \nu_{\bar{n}}\left(\boldsymbol{x}_{t}\right)\right\}$ have a unique vector $\boldsymbol{h}^{*} \in \mathbb{R}^{M_{n}(K)-d}$ in its orthogonal complement. Such a vector can be computed as the equilibrium point of the recursion

$$
\begin{aligned}
\hat{\boldsymbol{h}}_{t+1} & =\left(I_{M_{\bar{n}}(K)-d}-\mu \frac{\Gamma_{d} \Pi_{\bar{n}} \nu_{\bar{n}}\left(\boldsymbol{x}_{t}\right) \nu_{\bar{n}}\left(\boldsymbol{x}_{t}\right)^{\top} \Gamma_{d}^{\top}}{1+\mu\left\|\Gamma_{d} \Pi_{\bar{n}} \nu_{\bar{n}}\left(\boldsymbol{x}_{t}\right)\right\|^{2}}\right) \hat{\boldsymbol{h}}_{t} \\
\hat{\boldsymbol{b}}_{t} & =\frac{D \nu_{\bar{n}}^{\top}\left(\boldsymbol{x}_{t}\right) \Gamma_{d}^{\top} \hat{\boldsymbol{h}}_{t}}{e_{K}^{\top} D \nu_{\bar{n}}^{\top}\left(\boldsymbol{x}_{t}\right) \Gamma_{d}^{\top} \hat{\boldsymbol{h}}_{t}} .
\end{aligned}
$$

The vector $\boldsymbol{h}^{*}$ can be used to define a polynomial of degree $\bar{n}$ in $\boldsymbol{z}, p_{\bar{n}}(\boldsymbol{z})=\nu_{\bar{n}}(\boldsymbol{z})^{\top} \Gamma_{d}^{\top} \boldsymbol{h}^{*}$, that vanishes at $\boldsymbol{x}_{t}$ for all $t \geq 0$. Therefore, if there exist $\rho_{1}, \rho_{2}>0$ such that for all

\footnotetext{
${ }^{3}$ This is because there could be more than one normal vector $\boldsymbol{b}_{i}$ which is orthogonal to all regressors $\boldsymbol{x}_{t}$ associated with the $i$ th mode.
}

$j \geq \max \left\{\bar{n}_{a}, \bar{n}_{c}\right\}$ we have that

$\rho_{1} I_{M_{n}(K)-d} \prec \sum_{t=j}^{j+S} \Gamma_{d} \Pi_{\bar{n}} \nu_{\bar{n}}\left(\boldsymbol{x}_{t}\right) \nu_{\bar{n}}^{\top}\left(\boldsymbol{x}_{t}\right) \Pi_{\bar{n}}^{\top} \Gamma_{d}^{\top} \prec \rho_{2} I_{M_{n}(K)-d}$,

then $p_{\bar{n}}(\boldsymbol{z})$ must factor as a product of $\left(\boldsymbol{b}_{1}^{\top} \boldsymbol{z}\right) \cdots\left(\boldsymbol{b}_{n}^{\top} \boldsymbol{z}\right)$ with some polynomial $q_{\bar{n}-n}(\boldsymbol{z})$ of degree $\bar{n}-n$.

As before, except for a zero-measure set of initial conditions, the factorizability of $p_{\bar{n}}$ guarantees that its derivatives give the desired parameter vectors. The only problem is that the value of $d$ is not known beforehand, and so we must search for $d$ in an automatic fashion. One possible way of doing this is as follows

1) Set $d=0$.

2) Run two identifiers $\left(\hat{\boldsymbol{h}}_{t}^{1}, \hat{\boldsymbol{b}}_{t}^{1}\right)$ and $\left(\hat{\boldsymbol{h}}_{t}^{2}, \hat{\boldsymbol{b}}_{t}^{2}\right)$ as in (29)(30) for the current value of $d$ starting at two different initial conditions $\hat{\boldsymbol{h}}_{0}^{1} \neq \hat{\boldsymbol{h}}_{0}^{2}$, and wait until the two identifiers converge.

3) If the two identifiers converge to the same value, i.e. if $\hat{\boldsymbol{h}}_{t}^{1}-\hat{\boldsymbol{h}}_{t}^{2} \rightarrow \mathbf{0}$, then return either of the identified ARX model parameters, e.g. return $\hat{b}_{t}^{1}$.

4) If the two identifiers converge to two different values, but $\hat{\boldsymbol{b}}_{t}^{1}-\hat{\boldsymbol{b}}_{t}^{2} \rightarrow \mathbf{0}$, then set $\bar{n}=\bar{n}-1$ and go to 2 ).

5) If $\hat{\boldsymbol{b}}_{t}^{1}-\hat{\boldsymbol{b}}_{t}^{1} \nrightarrow \mathbf{0}$, then set $d=d+1$ and go to 2 ).

Note that, in practice, determining when a vector has converged, or when a vector has converged to zero requires the user to specify some thresholds.

\section{EXPERIMENTS}

In this section, we present four experiments evaluating the performance of the proposed algorithm for different values of the number of models and the model orders, as shown in Table I. We perform our experiments on an SARX model

$$
y_{t}=a\left(\lambda_{t}\right) y_{t-1}+b\left(\lambda_{t}\right) u_{t-1}+w_{t-1}
$$

with a periodic switching sequence $\lambda_{t} \in\{1,2\}$ of period 20 seconds, input $u_{t} \sim \mathcal{N}(0,1)$, noise $w_{t} \sim \mathcal{N}\left(0, \sigma^{2}\right)$, and parameters $a(1)=-0.9, a(2)=0.7, c(1)=0.8$ and $c(2)=$ -1 , so that $\boldsymbol{h}=[-0.8,1.46,-0.2,-0.63,-0.2,1]^{\top} \in \mathbb{R}^{6}$. We set the parameter of the recursive identifier to $\mu=1$.

We first perform our experiments in the absence of noise. In experiment 1 , both the number of modes and the model orders are correct. The hybrid model parameters and the ARX model parameters converge to their true values in only 100 seconds, as shown by the top three plots of Figure 1.

In experiment 2 , the number of modes is over estimated as $\bar{n}=4>2$, but the model orders are correct. From the last five plots of Figure 1, note that after approximately 60

TABLE I

EXPERIMENTAL PARAMETERS

\begin{tabular}{|c|c|c|c|c|c|c|}
\hline Experiment & $n$ & $n_{a}$ & $n_{c}$ & $\bar{n}$ & $\bar{n}_{a}$ & $\bar{n}_{c}$ \\
\hline 1 & 2 & 1 & 1 & 2 & 1 & 1 \\
2 & 2 & 1 & 1 & 4 & 1 & 1 \\
3 & 2 & 1 & 1 & 2 & 2 & 2 \\
4 & 2 & 1 & 1 & 3 & 2 & 2 \\
\hline
\end{tabular}



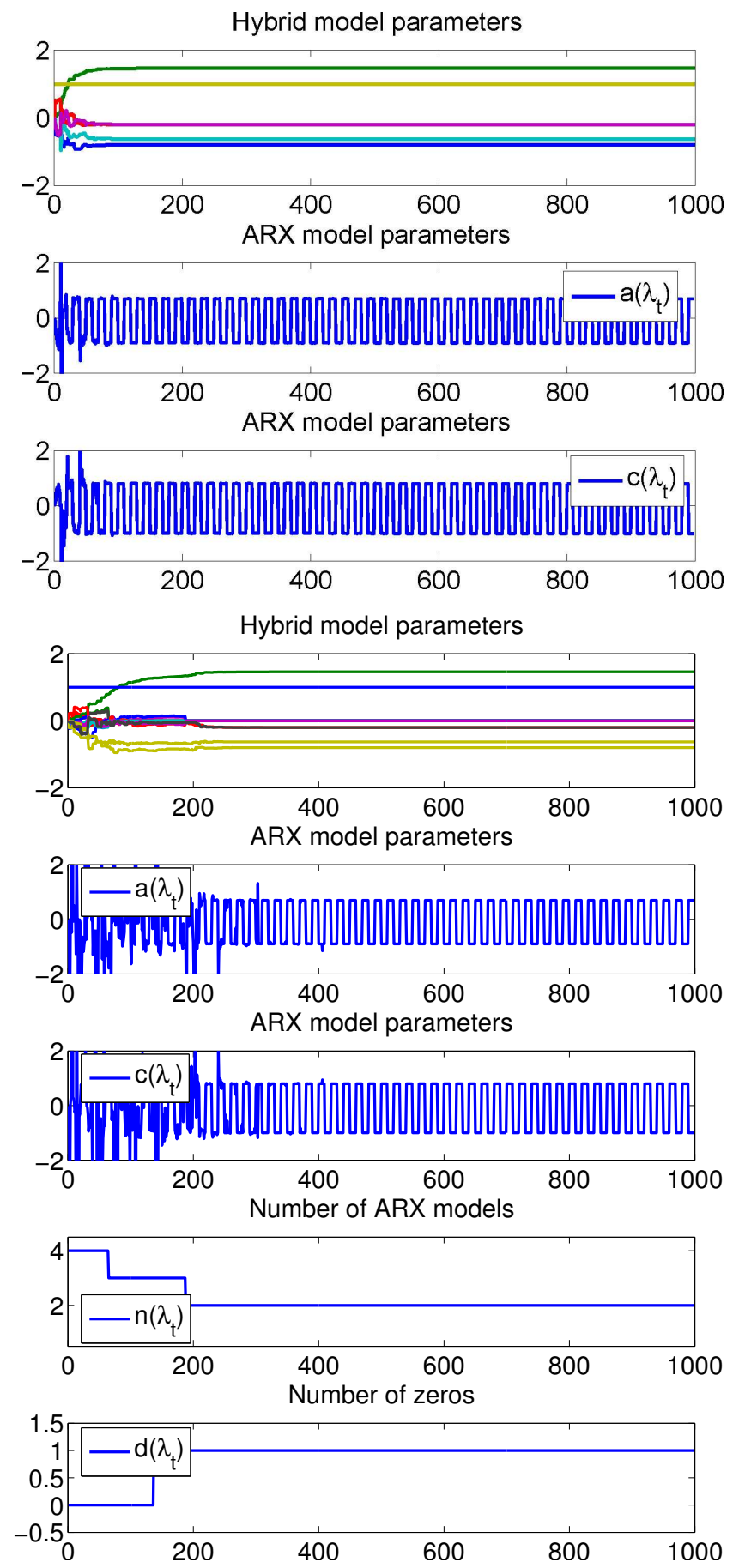

Fig. 1. Evolution and convergence of system parameters without noise for Experiments 1 (first three plots) and Experiment 2 (last five plots).

seconds the number of modes is reduced to 3. Afterwards, the hybrid model parameters converge faster than the ARX model parameters, causing the algorithm to increase the number of zeros. Once the ARX parameters of the two identifiers converge to the same value, the number of models is reduced to $\bar{n}=2$, which is the correct value. Overall, the parameters converge after approximately 420 seconds.

In experiment 3 , the number of modes is correct, but the model orders are overestimated. Notice from the top five plots of Figure 2 that after approximately 300 seconds the algorithm starts increasing the number of zeros to $d=3$. Since in this case any number of zeros $1 \leq d \leq 5$ guarantees convergence, after approximately 550 seconds, both the hybrid and the ARX model parameters have converged. Notice also how $a_{2}\left(\lambda_{t}\right)$ and $c_{2}\left(\lambda_{t}\right)$ converge to zero, showing the correct estimation of the model orders.

In experiment 4 , both the number of models and the model orders are overestimated. As shown by the last five plots of Figure 2, the hybrid model parameters converge faster than the ARX model parameters, hence after only 300 seconds the algorithm starts increasing the number of zeros. Once the ARX model parameters of the two identifiers converge to the same value (approximately at $t=1,015$ seconds), the algorithm reduces the number of modes to $\bar{n}=2$, the correct value. Around $t=1,250$ seconds, all ARX model parameters converge to their true values. The system orders are also correctly estimated.

Figure 3 shows the evolution of the model parameters for experiments 3 and 4, this time with a noise level of $\sigma=0.01$. The algorithm works in the presence of noise; however, as expected, its performance deteriorates.

\section{CONCLUSIONS AND FUTURE WORK}

We have presented a recursive algorithm for identifying the parameters of switched ARX models with unknown number of modes and unknown and possibly different orders and derived persistence of excitation conditions on the embedded input/output data that guarantee the exponential convergence of the identifier. It remains open to determine persistence of excitation conditions on the input and switching sequences only, and to extend this approach to multivariate systems in state space representation.

\section{REFERENCES}

[1] B.D.O. Anderson, R.R. Bitmead, C.R. Johnson Jr., P.V. Kokotovic, R.L. Ikosut, I.M.Y. Mareels, L. Praly, and B.D. Riedle. Stability of Adaptive Systems. MIT Press, 1986.

[2] A. Bemporad, A. Garulli, S. Paoletti, and A. Vicino. A greedy approach to identification of piecewise affine models. In Hybrid Systems: Computation and Control, LNCS, pages 97-112. 2003.

[3] A. Bemporad, J. Roll, and L. Ljung. Identification of hybrid systems via mixed-integer programming. In IEEE Conf. on Decision \& Control, pages 786-792, 2001.

[4] G. Ferrari-Trecate, M. Muselli, D. Liberati, and M. Morari. A clustering technique for the identification of piecewise affine systems. Automatica, 39(2):205-217, 2003.

[5] J. Harris. Algebraic Geometry: A First Course. Springer-Verlag, 1992.

[6] A. Juloski, S. Weiland, and M. Heemels. A Bayesian approach to identification of hybrid systems. In IEEE Conf. on Decision \& Control, 2004

[7] Y. Ma and R. Vidal. Identification of deterministic switched ARX systems via identification of algebraic varieties. In Hybrid Systems: Computation and Control. 2005.

[8] R. Vidal. Identification of PWARX hybrid models with unknown and possibly different orders. In Proceedings of the American Control Conference, 2004.

[9] R. Vidal and B.D.O. Anderson. Recursive identification of switched ARX hybrid models: Exponential convergence and persistence of excitation. In IEEE Conf. on Decision \& Control, 2004.

[10] R. Vidal, S. Soatto, Y. Ma, and S. Sastry. An algebraic geometric approach to the identification of a class of linear hybrid systems. In IEEE Conf. on Decision \& Control, 2003. 
Hybrid model parameters
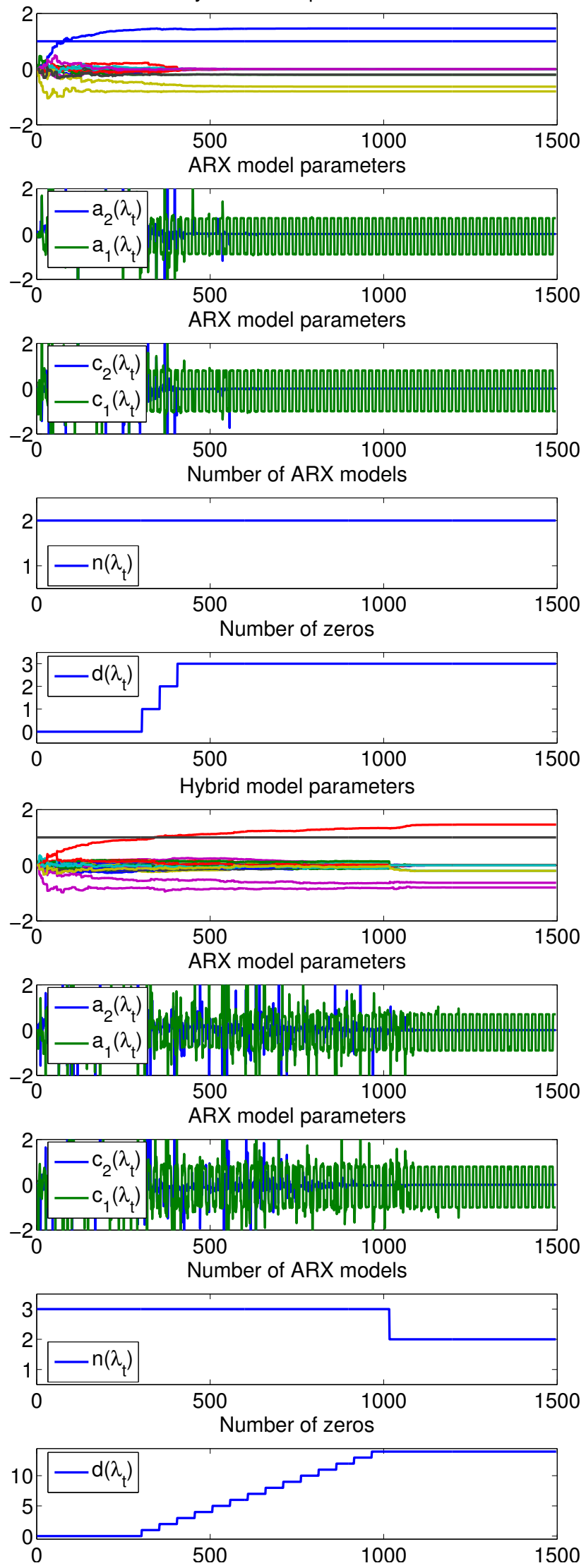

Fig. 2. Evolution and convergence of system parameters without noise for Experiment 3 (first five plots) and Experiment 4 (last five plots).
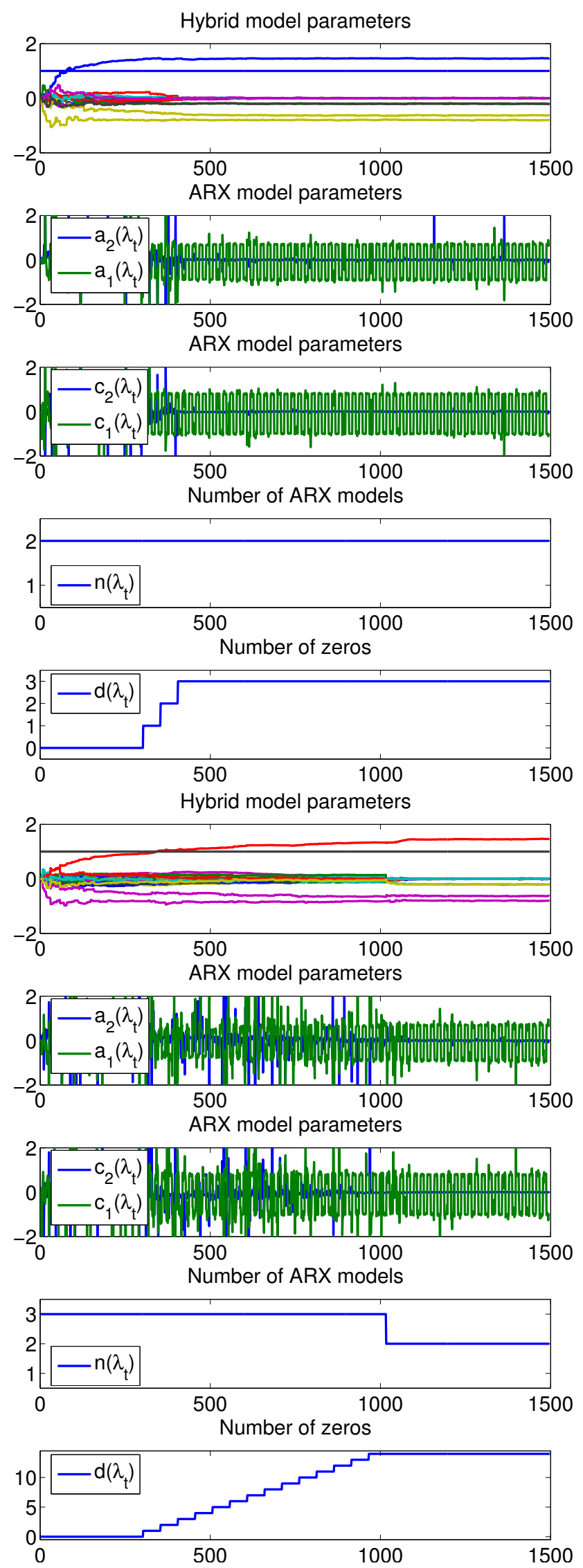

Fig. 3. Evolution and convergence of system parameters with $\sigma=0.01$ for Experiment 3 (first five plots) and Experiment 4 (last five plots). 